\title{
External cervical resorption: Radiological diagnosis and literature (Review)
}

\author{
ROXANA-MARIA TALPOS-NICULESCU ${ }^{1 *}$, LUMINITA-MARIA NICA $^{1^{*}}$, MALINA POPA ${ }^{2 *}$, \\ SERBAN TALPOS-NICULESCU ${ }^{3}$ and LAURA CRISTINA RUSU ${ }^{4}$
}

\author{
${ }^{1}$ Third Department, Discipline of Restorative Dentistry and Endodontics, Research Center TADERP, \\ Faculty of Dental Medicine, ${ }^{2}$ Second Department, Discipline of Pedodontics, Pediatric Dentistry Research Center, \\ Faculty of Dental Medicine, 'Victor Babes' University of Medicine and Pharmacy of Timisoara, 300070 Timisoara; \\ ${ }^{3}$ Second Department, Discipline of Oral and Maxillo-Facial Surgery, Faculty of Dental Medicine, \\ 'Victor Babes' University of Medicine and Pharmacy, 300062 Timisoara; ${ }^{4}$ First Department, Discipline of Oral Pathology, \\ Multidisciplinary Center for Research, Evaluation, Diagnosis and Therapies in Oral Medicine,
}

Faculty of Dental Medicine, 'Victor Babes' University of Medicine and Pharmacy of Timisoara, 300174 Timisoara, Romania

Received May 31, 2021; Accepted June 30, 2021

DOI: $10.3892 / \mathrm{etm} .2021 .10499$

\begin{abstract}
External cervical resorption (ECR) is a relatively unknown and insidious pathology characterized by the loss of hard dental tissues such as: Enamel, cementum and dentine due to clastic function. It begins as a localized resorptive process that initiates on the area of the root beneath the epithelial attachment and the coronal part of the alveolar process, involving vital and non-vital tissues. Despite the fact that there are several potential predisposing factors related to ECR, its aetiology still remains poorly understood and more research is needed to establish the cause-and-effect relationship of all the etiological factors. Improved radiographic detection using cone-beam computed tomography (CBCT) is required in order to correctly classify and assess this entity. This provides a three-dimensional insight into the lesion, regarding the location, the size, the depth and the circumferential spread of the ECR defect. It also allows establishment of the most efficacious treatment plan and management. The purpose of this literature review is to cover the relevant literature concerning the etiology, pathogenesis, clinical and radiological presentation and management of ECRs (based on the CBCT findings).
\end{abstract}

Correspondence to: Dr Serban Talpos-Niculescu, Second Department, Discipline of Oral and Maxillo-Facial Surgery, Faculty of Dental Medicine, 'Victor Babes' University of Medicine and Pharmacy, 5 Take Ionescu Boulevard, 300062 Timisoara, Romania E-mail: talpos@yahoo.com

"Contributed equally

Key words: external cervical resorption, cone beam computed tomography, dentinal penetration, circumferential spread, external approach, internal approach

\section{Contents}

1. Introduction

2. Pathogenesis and etiology

3. Histology

4. Classification

5. Clinical and radiographic presentation

6. Treatment

7. Conclusions

\section{Introduction}

Dental resorption is considered to be a challenge to dentistry due to its complexity. Root resorption (RR) represents a pathological process that causes the loss of cementum, dentine and/or enamel, almost irreversibly, concerning vital and non-vital teeth, as a result of odontoclastic function (1). Although root resorption is necessary in temporary dentition enabling the eruption of the permanent successors (physiological root resorption), root resorption of permanent teeth is unfavorable as it may lead to perpetual injury, which might require dental treatment or, sometimes, extraction (2).

Generally, it can simply be classified as internal or external resorption, depending on the location on the root surface. External root resorption can be further subclassified into surface resorption, external inflammatory resorption, external replacement resorption, external cervical resorption, transient apical resorption (3-6). One of the least understood types of external resorption is external cervical resorption.

External cervical resorption (ECR) is a clinical term used to define a quite uncommon, insidious and often aggressive form of external root resorption, which can appear in any permanent tooth (5). ECR is a dynamic and pathological process characterized by its cervical position on the tooth, arising immediately below the epithelial attachment and the coronal part of the bone, this zone being named the zone of 
the connective tissue attachment (5). Its nature is an extremely aggressive one, concerning periodontal, dental and in later phases pulpal tissues, causing substantial damage to the tooth structure (7).

Other various terms used to describe ECR are invasive cervical resorption, odontoclastoma, idiopathic external resorption, fibrous dysplasia of the teeth, burrowing resorption, late cervical resorption, extra-canal invasive resorption, peripheral cervical resorption, supra-osseous inflammatory root resorption, subepithelial inflammatory resorption, and periodontal infection resorption $(6,8)$.

In the present article, the term used to describe this entity will be external cervical resorption (ECR), as it reveals exactly the starting point of the resorptive process on the tooth surface.

Electronic literature databases (PubMed and Web of Science) were searched to identify relevant studies related to ECR. The inclusion criteria were reviews, case reports, case series, clinical studies, clinical trial and histological studies up until 2020. Of the 121 studies identified from the electronic PubMed's database search, 62 studies were included for this review. The electronic Web of Science's search revealed 182 articles, from which only 19 were reviews. The review of the literature demonstrated that there are few original scientific articles on this topic. Most of the articles were case reports, case series, describing the possible etiology and the possible therapeutic options regarding ECR.

On the other hand, few articles on histopathology and diagnosis were found in the literature, leading to poor diagnosis, under-reported and mismanagement of this entity (2).

\section{Pathogenesis and etiology}

ECR is a dynamic and complex process. The process of external resorption can be described by three main phases: The initiation of resorption, expansion of resorption and the reparative stage $(2,7,9)$.

The resorption initiation phase is symbolized by disruption of the periodontal ligament (PDL) and by damage of the protective unmineralized cementum (10). This process leads to a localized inflammatory response of the exposed dentine so macrophages can migrate to the inflamed area, causing the formation of granulation tissue, which can link to the exposed dentine. The exposed dentine is susceptible to resorption from the adjacent bone or to immune cells (2). Three circumstances are required in order for cervical resorption to take place: Presence of blood supply, recruitment of circulating cells and lack of protective layer $(5,11)$. Some studies suggest that hypoxia can lead to activation of osteoclastic phenomena by disturbing the metabolism and interfering with the recovery of fibroblasts $(2,12)$.

After starting from its location, the resorption extends in a circumferential and apicocoronally direction around the root canal, invading the tooth structure by resorbing cementum, dentine and enamel $(2,11,13)$. The pulpal tissue remains vital, being protected by the thin pre-dentine layers so the resorptive defect does not perforate into the root canal $(2,7,13)$. Wedenberg and Lindskog suggest that an inhibitor of macrophage propagation is included in the organic component of dentine, making the pre-dentine and dentine resilient to resorption (14). These changes may be the result of various stimulating factors such as: Infection (microorganisms), continuous and discontinuous mechanical force on PDL (orthodontic treatment, parafunction), which can lead to hypoxia $(2,12,14)$.

Resorption and repair can take place in parallel in different parts of the same lesion. Osteoblast-like cells can form bone-like tissue that can penetrate into the resorptive defect. This is thought to be a form of healing $(2,7)$.

Regarding the involvement of bacteria, two mechanisms can be described for the ECR lesions. The first one proposes that microorganisms are not essential to the initiation of ECR but may appear as secondary aggressors to maintain the resorptive process. After the loss of protective unmineralized tissue, clastic cells moving in the subepithelial connective tissue area connect to the root. The bacteria from the periodontal pocket that may invade the lesion can promote and maintain the inflammatory resorptive process. According to the second mechanism, microorganisms are vital to the beginning and propagation of ECR. Loss of protective unmineralized tissue (pre-cementum) with simultaneous inflammation produced by microorganisms in the periodontal pocket originates the resorptive development at the cervical area of the ECR tooth. Bacteria in the lesion participate in the perpetuation of this resorptive process $(7,8,15,16)$.

Recent histological findings seem to indicate that bacteria are not essential for the initiation of the defect. It appears that microorganisms colonize the resorptive process in later stages of the resorption, based on the observation that microorganisms are identified only at the outer layers of the defects $(7,15,16)$.

Research indicates a lack of clarity surrounding the etiology of this condition. The etiology of ECR remains unclear, although several potential predisposing factors have been identified and associated with this condition $(2,9)$. Orthodontic treatment, previous history of traumatic injury (luxation, avulsion), restorative and endodontic procedures, internal bleaching using hydrogen peroxide $30 \%$ and dentoalveolar surgery are the principle potential predisposing factors for ECR (2,6,9,17-19).

Based on a study by Heithersay, in which 257 ECR teeth were analyzed, orthodontic treatment $(28.4 \%)$ was the most common factor and previous traumatic injury was the second one (17). Additional factors such as internal bleaching, surgery and restorative treatment were identified as predisposing factors (2,17). According to Mavridou et al, who assessed 337 ECR teeth, several additional predisposing factors were reported (19). These included: Malocclusion, parafunctional habits, poor oral hygiene, extraction of an adjacent tooth, impacted teeth, eruption disorders (pressure generated by canine eruption on lateral incisors), viral etiology, individual genetic propensity and playing wind instruments (19). Orthodontic treatment as a predisposing factor increased from 28.4 to $45.7 \%(17,19)$.

Any surgical procedure that injures the cervical region (cementoenamel junction) can be considered an etiological factor for ECR (5). Extraction of an adjacent tooth that led to damage of the cervical area of a neighboring tooth, may result in ECR (20). Fuss et al postulated that impacted teeth such as third mandibular molars could cause resorptive lesions affecting mandibular second molars (21). Sometimes, spontaneous resorption of impacted tooth can occur. Orthognathic surgery can be considered as a predisposing factor for ECR; 
heat damage to bone, impairment of blood supply are important predisposing factors associated with root resorption $(17,19)$. Lateral incisors can suffer cervical resorption lesions due to the pressure exerted by canine eruption $(22,23)$.

Both Heithersay's and Mavridou's studies have shown that internal bleaching can be a possible predisposing factor of ECR $(17,19)$. The $30 \%$ hydrogen peroxide $\left(\mathrm{H}_{2} \mathrm{O}_{2}\right)$ liberated during internal bleaching presents permeability through dentinal tubules and moves out through the dentine gaps in amelodentinal junction, which may denature the organic components of dentine, cementum, provoking a foreign body reaction near cementoenamel junction (CEJ). This immunological response results in activation of phagocytes and attachment of clastic cells, which can lead to the initiation of the resorptive process $(17,19)$. An acidic environment caused by bleaching paste may enhance the osteoclastic activity leading to ECR (24-26). While Heithersay reported internal bleaching as a potential predisposing factor in $13.6 \%$ of cases (17), Mavridou et al postulated that only $2.7 \%$ of ECR cases appear due to internal bleaching (19). The lower percentage of appearance of internal bleaching as a predisposing potential factor in Mavridou's study may be due to softer bleaching products that are currently used (19). However, patients should be advised of the potential risk for ECR $(2,19)$.

Recently, ECR has been associated with viral etiology. ECR also occurs in domestic, captive and wild cats and the pathology is known in the veterinary literature as feline dental resorptive lesions, feline odontoclastic resorptive lesions (FORL), tooth resorption (TR), or neck lesions $(27,28)$. Von Arx et al indicated that feline herpes viruses (FHV) can be transmitted to humans and may have a relevant position in the pathogenesis of human cervical resorptive lesions (29). Nevertheless, future studies are necessary to confirm the likelihood of viral transmission from cats to humans and the role of FORL/TR in cervical lesions.

Teeth presenting with previous traumatic injury episode such as concussion or avulsion can lead to rupture/damage of PDL, which may be a cause for resorptive lesions $(10,17,19)$. Mavridou et al and Gunst et al described cases of ECR in individuals who play wind instruments $(19,30)$. Apart from these, systematic factors, poor oral health, nutrition, and an individual's genetic propensity can be associated factors for ECR $(11,17,19)$.

A recent study of Irinakis et al based on a 10-year observation of endodontic patients showed a prevalence of ECR of $2.3 \%$, in which predisposing factors were identified in approximately $78 \%$ patients with ECR defects (31).

Therefore, the exact etiology of ECR has not been confirmed, because in the majority of cases, more than one predisposing factor has been identified, showing that ECR is a multifactorial entity and not idiopathic (17,19,31). However, more research is needed to establish the cause-and-effect relationship of all of these suggested etiological factors.

\section{Histology}

ECR is a complex and dynamic process. According to Mavridou et al, ECR cases share several common characteristics such as: Initiative resorption phase (portals of entry), propagation resorption phase in which channels and external interconnections are found (portals of exit), the presence of the so-called pericanalar resorption-resistant sheet (PRRS), the reparative phase characterized by replacement of the resorbed tissue with bone-like tissue and the remodelling phase of the repaired tissue (7).

The initiation point is characterized by the so-called portal of entry, which is located at the level of the cement, under the epithelial attachment. In order to occur, ECR requires the presence of localized damage and/or removal of the periodontal ligament from the entry orifices. This area is described by the presence of connective tissue with lympho-plasma infiltrates and an increase in bone-like tissue with the fusion of the neighboring alveolar bone to dentine $(2,7)$.

The resorptive area described by channels and external interconnections, starts from the initiation point (portals of entry) and extends three-dimensionally, being able to encircle or progress towards the pulpal tissue, resulting in destruction of dental tissues (dentin, enamel, cement). In this way, certain channels and interconnections with the periodontal ligament are created, the so-called portals of exit $(2,7)$.

Mavridou et al and Gunst et al, based on histological and micro-CT findings, suggested that PRRS, an uneven layer located around the root canal, which is characterized by dentine and, occasionally, by bone-like tissue, may function as a protective film against perforation of the root canal against the resorptive lesion $(7,13,20)$. PRRS resistance to resorption can be attributed to the low mineral content that inhibits the attachment of clastic cells as well as normal oxygen tension from blood supply in the pulp tissue that decreases clastic action (7).

In vitro studies of Wedenberg and Lindskog demonstrated that an inhibitor of macrophage spreading is present in the organic components of dentin and that this inhibitor may be responsible for the resistance of pre-dentine to resorption (14). However, in later and advanced stages, pulp damage can take place (7).

There are some differences between endodontically treated and untreated teeth, these differences being related to the presence of PRRS. The intensity of resorption was observed to be higher in endodontically treated teeth, an aspect associated with the removal of this protective layer during endodontic treatment, which involves chemo-mechanical preparation (15). Moreover, the loss of pulpal vitality can induce a hypoxic environment that can stimulate the continuation of clastic activity. The apposition of bone-like tissue into the resorptive defect is more common in vital teeth $(7,15)$. The hypoxic environment, generated by the excision of the pulpal tissue can be a factor for the constant growth of the granulomatous tissue, thus inhibiting osteoblast formation and encouraging the osteoclastic process to occur (32-34). A non-toxic environment is preserved throughout the PRRS, allowing the substitution of granulomatous tissue from the resorptive defects with bone-like tissue during the repair and remodelling stages (7). Repair of the resorbed tissue takes place by apposition of reparative bone-like tissue through portals of entry into the tooth. The histological findings of Mavridou et al showed bone-like related cells (osteoblast-like cells, osteocytes) and osteoid tissue (7).

Cyclic resorption and reforming of the bone-like tissue containing clastic and blastic cells are the main activities that 
describe the last phase called the remodelling phase. It is likely that in various regions of the same tooth, resorption of dentine, active repair and remodelling of the bone-like tissue can occur together (7).

\section{Classification}

Heithersay developed the first classification of ECR in 1999 (17). This classification is based on periapical radiographs, being a two-dimensional one. Heithersay divides ECR lesions into 4 classes, depending on the size and extent of the resorption process in the dentine: Class 1 , a small lesion in the cervical area of the tooth, with a slight penetration into the dentine; Class 2, a deeper penetration of the defect towards the pulp chamber, with little or no involvement of the root dentin; Class 3, a deep cervical resorbable lesion, affecting the root dentin of the coronal third part; Class 4 is characterized by an extensive resorptive defect beyond the coronal third of the root dentine (17). Unfortunately, this classification, being a two-dimensional one, based on the interpretation of periapical radiographs, does not provide accurate information concerning the circumferential damage, the depth of the defect, the surface on which the lesion is located (buccal or palatal), the proximity of the lesion to the root canal or the true phase in which the lesion is found (reparative or resorptive) (35). This bi-dimensional interpretation does not provide an accurate assessment of this entity (as observed in Fig. 1) and may lead to incorrect establishment of treatment and management plans.

Recently, Patel et al described a new three-dimensional classification of ECR based on CBCT interpretation (18). This new classification takes into account three parameters, namely: The height of the lesions, the circumferential spread and the proximity to the pulpal tissue. The score for the height of the lesions is conducted as follows: Score ' 1 ' is given for the supra-crestal defects, ' 2 ' for sub-crestal ones, ' 3 ' for those extending in the middle third of the root and ' 4 ' for defects that extend to the apical area of the root. Regarding the circumferential spread, the lesions that do not exceed $90^{\circ}$ are noted with 'A'; 'B' are those that are found between $90^{\circ}$ and $180^{\circ}$; ' $\mathrm{C}$ ' indicates lesions larger than $180^{\circ}$ but smaller than $270^{\circ}$ and ' $D$ ' indicates defects that exceed $270^{\circ}$. The third parameter, the proximity to the root canal is noted with the small letter ' $\mathrm{d}$ ', which shows that the lesion is confined with dentine and with the letter ' $p$ ' if the lesion perforates the root canal (18). Fig. 2 shows an example of a Class 2Bp lesion diagnosed on CBCT.

The maximum height, circumferential spread and depth of the lesion are noted after performing and interpreting periapical radiographs and $\mathrm{CBCT}$ scans, thus resulting in a three-dimensional gradation of the ECR lesion $(18,36)$. It is recommended that the ECR resorptive lesions be diagnosed and assessed with the help of CBCT before establishing a treatment plan as indicated by the American Association of Endodontists (AAE)/American Academy of Oral and Maxillofacial Radiology Joint Position Statement (AAOMR) and European Society of Endodontology Position Statement (ESE) $(36,37)$.

A three-dimensional classification of ECR is necessary and relevant, because in vivo CBCT studies, ex vivo micro-CT and nano-CT have demonstrated the complexity of ECR invasion (portal of entry, small channels and their interconnections with
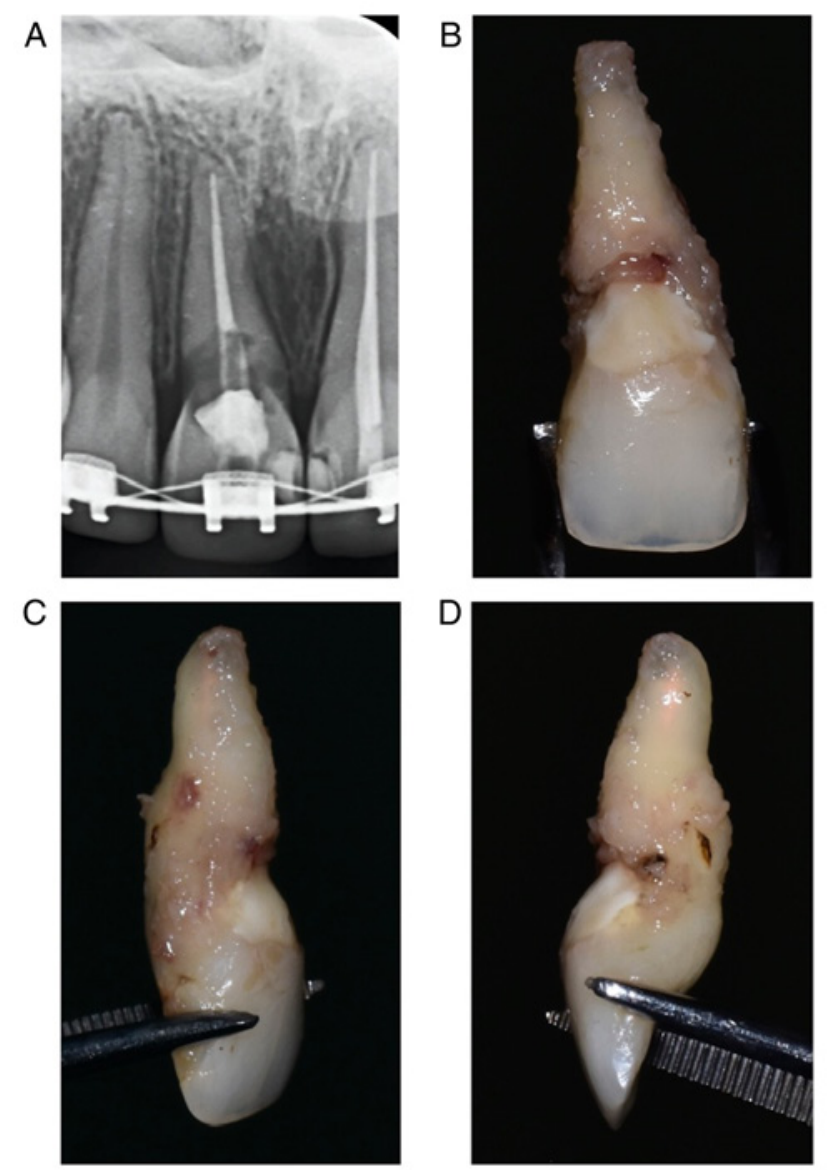

Figure 1. Radiological and clinical findings of tooth 11 with external cervical resorption (ECR). The tooth diagnosed with Class 3 ECR according to Heithersay's bi-dimensional classification was extracted due to failure of the conservative treatment. (A) The periapical radiography shows the extension of the resorptive lesion in the coronal third of the root, and the endodontic treatment performed prior to the external repair of the lesion, without the possibility to evaluate the lateral spread of the lesion and its depth. (B) Buccal view of the extracted tooth with the lesion repaired with glass ionomer cement. (C and D) From mesial and distal view, the circumferential spreading of the lesion and also its depth was able to be observed.

the periodontal ligament), an invasion that can be different in all three dimensions $(7,20,38)$.

This provides a three-dimensional insight into the lesion and the capacity to correctly assess the entity and to establish the most proper treatment plan and management.

\section{Clinical and radiographic presentation}

Clinically, there is no classic presentation or particular symptomatology for ECR. The lesion often begins asymptomatically, insidiously, being accidentally discovered at a clinical and/or radiological routine check-up (8). This is characteristic for the initial lesions, discovered in early stages. In more advanced cases, when the defect is in the proximity of the pulp chamber or even perforates it, patients may report symptoms similar to reversible or irreversible pulpitis (temperature sensitivity) and/or symptoms similar to apical periodontitis (percussion pain, fistula) $(1,8)$. In initial stages, vitality tests are within normal limits, as long as there is no involvement of the pulp and the pulp has not become necrotic (1). 


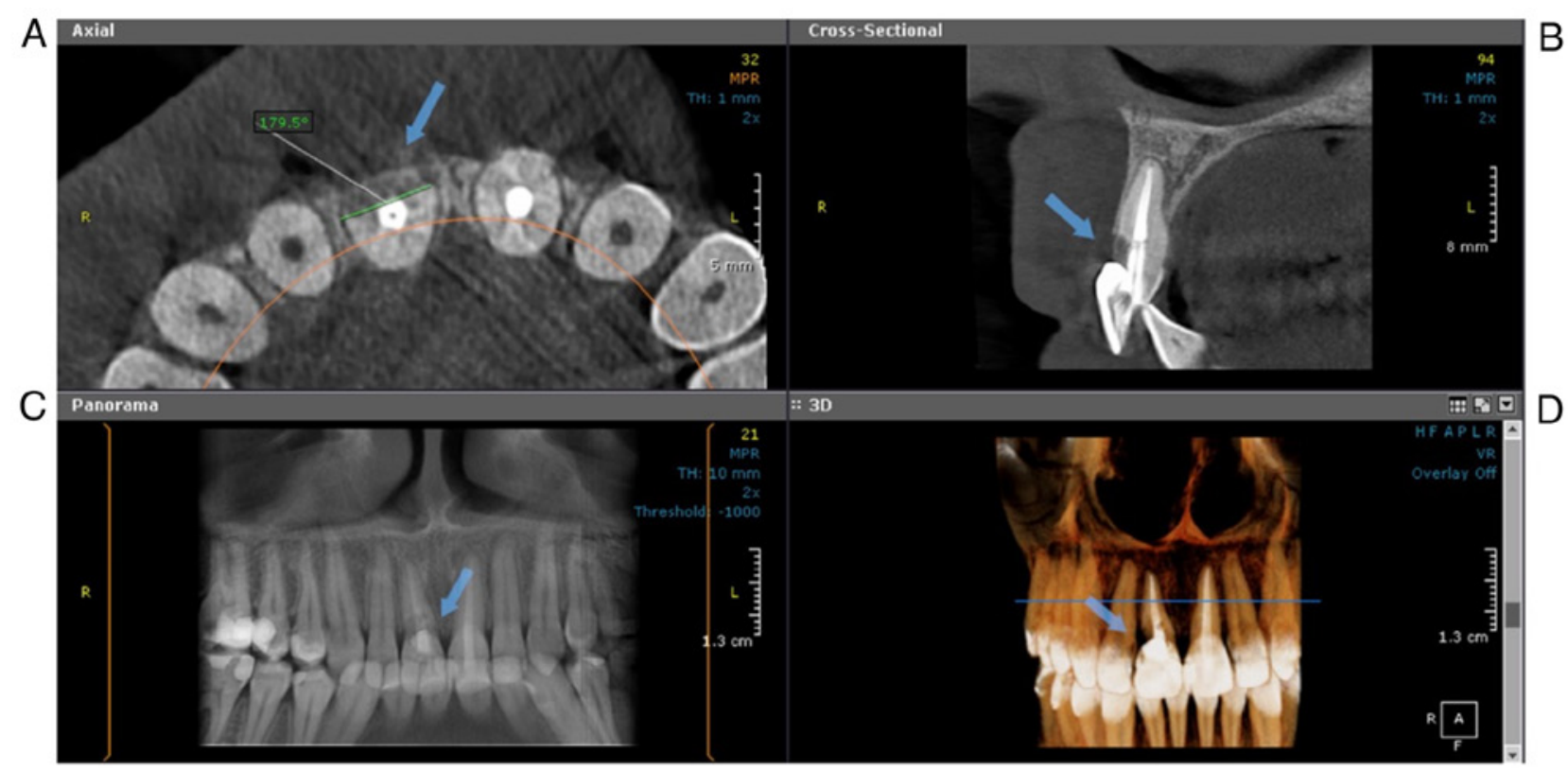

Figure 2. Cone beam computed tomography (CBCT) of tooth 11 with external cervical resorption (ECR). On the CBCT, the external cervical resorption can be more accurate diagnosed and classified as Class $2 B p$ according to Patel's three-dimensional (3D) classification. (A) On the axial section, the lateral extension of the ECR lesion can be easily observed and measured as spreading almost on half of the root circumference $179^{\circ}$ (score B). (B) In the cross-sectional view, the penetration of the lesion into the root canal and also its extension below the alveolar crest can be assessed and measured (score 2p). (C) On the panoramic view, no data regarding the depth, circumferential spreading and dentinal penetration can be specified. (D) The 3D reconstruction allows to evaluate the lateral spread and the extension of the lesion into the root canal (blue arrows mark the ECR lesion on all sections).

This is why the diagnosis of ECR is challenging, and the differential diagnosis with cervical defects produced by caries or cavities is difficult to achieve, but this aspect is of a tremendous importance in establishing a correct treatment plan. Resorptive defects of different etiology can be assessed or diagnosed using periodontal probes or periodontal scalers. The defects determined by caries lesions are soft and sticky on probing, due to disintegration of the organic component of the dentin $(1,3,39)$. Moreover, gingival recession may occur (40). On the other hand, the cavities resulting from ECR are hard and scratchy on probing, giving a feeling of mineralized tissue. In addition, the edges are sharp, like a knife $(1,39)$.

A pink spot can be observed in the cervical third area of the ECR teeth, being a pathognomonic sign of this entity $(3,8)$. This is due to the high vascularity of the granulomatous tissue, which can be visible through the thin enamel in the cervical area. The presence of granulomatous tissue is also the reason why bleeding is observed during probing $(1,39)$. However, the appearance of pink spots is quite rare. Teeth with ECR may also have dyschromia due to mortification of the pulpal tissue. In this case their color will be grey (2).

From a radiological point of view, the lesions can be symmetrical or asymmetrical. Their margins can vary from well-defined and smooth, to poorly defined and rough, or with no clear limits between the lesion and the healthy dental tissues (41). ECR may appear radiolucent (if the lesion is identified in its active resorption phase), radiopaque (if the lesion is detected in the repair phase as a result of ossification of the granulomatous tissue) or there may be combinations of both phases (the lesion appearing radiopaque and radiolucent) $(7,20)$.

In addition, ECR lesions can be misdiagnosed as internal resorption (IR). In ECR without perforation, the walls of the root canal should be intact, and the entire contour of the root canal must be observed, with the lesion located on the lateral surface of the root. In IR, the contour of the root canal is enlarged, but no defect is observed on the outer surface of the root, if the lesion did not complicate with a perforation or root fracture (42).

In order to avoid establishing an erroneous diagnosis of IR, multiple periapical radiographs can be performed from various angles. In the case of ECR, the lesion will look as if it is moving with the change of horizontal angle of the X-ray tube. The lesions located buccally move in the opposite direction, while those located lingually move in the same direction with the parallax shift. This can also help to determine the location of the lesion when clinically it is not possible (41).

However, periapical radiographs (PRs) have some disadvantages: The true extent of the lesion can be distorted which can result in underestimating or overestimating the size of the lesion. Being a two-dimensional image, only the height and width of the lesion can be analysed; details concerning depth and circumferential damage are minimal $(35,43)$. All of these disadvantages can lead to misdiagnosis and wrong management of the lesion.

Rodriguez et al established that the use of CBCT could lead to a more accurate diagnosis, assessment and/or management of difficult endodontic cases $(44,45)$.

The use of CBCT has exceeded the limits of bi-dimensional radiographs; the true nature of the lesion, size, circumferential extension and proximity to the root canal can be identified (13).

The European Society of Endodontology (ESE) also underlined the relevance and importance of using CBCT for the management of potential ECR lesions in 2014, as well as the AAE/AAOMR in 2015 (36,37).

The efficiency in the detection and classification of ECR was studied by Vaz de Souza et al in an ex vivo study, using 
and comparing two different CBCT scanners, Kodak and Morita with periapical radiographs (PRs) (43). Examiners were asked to locate and identify the tooth surface area/areas on which simulated lesions of ECR were located, by analyzing PRs and CBCTs. They correctly identified the lesion's location on the tooth surface in $87.8 \%$ with the KODAK CBCT scanner, in $89.1 \%$ with MORITA CBCT, and in only $49.4 \%$ on periapical radiographs. In addition, examiners were asked to classify the identified ECR lesions according to Heithersay's classification. Only on $32 \%$ PRs, lesions were correctly classified, compared to $70 \%$ for the MORITA CBCT, and $71.4 \%$ for KODAK CBCT (43).

Thus, the improved precision of CBCT has not only the advantage of a more accurate recognition, evaluation and classification of ECR, but helps also in the selection of the most suitable management plan.

\section{Treatment}

The final aim of the management strategy is to ensure that the tooth diagnosed with ECR is maintained in a healthy and functional status on the dental arch, avoiding tooth extraction, and to improve aesthetics when indicated.

In order to maintain the affected tooth on the dental arch, in a healthy and functional status, it is necessary to proper restore the tooth, by excavating the resorptive tissue, closing of the subsequent defect and portals of entry in order to arrest the resorptive process (9).

Performing a preoperative CBCT helps identify the true nature of the resorption, providing valuable information that can help establish a correct treatment plan $(9,18,35,46)$. Depending on the extent of the damage, the location of the lesion and its nature, two main therapeutic strategies can be proposed: Internal repair or/and external repair. In some of the cases, when the lesion has perforated the root canal walls or this may happen during the excavation of the resorptive process, endodontic treatment might be required as well $(16,46,47)$.

External approach can be combined with surgical treatment, such as flap surgery, when the lesions are extended far apically than the gingival margin. In cases where the resorptive lesions are located above/at the gingival margin level and are accessible to excavation, non-surgical repair and treatment can be the optimal solutions (16).

Small lesions, with inaccessible entry points which may extend apicocoronally and circumferentially around the root canal space, can be treated only using an internal approach, defined by the endodontic treatment and the restoration of the lesion $(7,46)$.

External approach. External repair of smaller resorptive processes, such as Heithersay class 1 and 2 or 3D Patel classes $1 \mathrm{Ad}, 2 \mathrm{Ad}, 2 \mathrm{Bd}$, is thought to have a positive outcome; in these cases, the root canal is typically intact and the pulpal tissue is healthy, without any symptoms of reversible or irreversible pulpitis $(18,47)$.

The external non-surgical approach can be performed on ECR teeth on which the lesions are located above the coronal third of the root, whereas the ECR lesions located below the gingival margin can benefit from surgical repair, including an intra-crevicular incision or muco-periosteal flap surgery $(47,48)$. The first external repair technique of ECR lesions was described by Heithersay and was characterized by topical application of $90 \%$ aqueous trichloroacetic acid solution (TCA) to the resorptive cavities, excavation of the infected tissue, endodontic treatment if the root canal was perforated and restoration with glass ionomer (GI) $(8,47)$.

The application of TCA is used in order to stimulate coagulation necrosis of the lesion and tissue located in an accessible channel of ECR (49). The TCA further penetrates the more inaccessible resorptive channels, which may not be visible and opened for excavation by mechanical instruments $(38,47)$. However, despite its advantages, TCA is very caustic, and attention must be paid when utilizing it because it can cause inflammation and possible irritation in neighboring tissues such as: Periodontal tissue, oral mucosa and/or skin. In order to protect the neighboring tissues, the rubber dam isolation system can be applied to the causal tooth. The TCA manipulation technique involves soaking a cotton pellet or micro-brush in the solution, then removing the excess and applying it in direct contact with the granulomatous tissue of the resorptive process for about 3-4 min, until coagulation necrosis occurs $(47,49)$.

The technique is very sensitive, and it necessitates the use of magnification, such as surgical microscope or loups, for the excavation of the granulomatous tissue with the help of hand instruments (excavators); the use of burs should be reduced to the maximum, in order to protect the intact remaining tooth structure (46). Small internal resorptive lesions can be accessed by using ultrasonic instruments under magnification without any risk of removing unnecessary sound dentine and can securely debride the lesions without damaging the periodontal tissues (16). The use of magnification helps to differentiate between healthy dentine and reparative hard tissues, and to remove as much as possible from the resorptive tissue. Being unable to completely remove the resorptive tissue, the lesions can recur (1).

If during the excavation of the ECR lesion the root canal is close to/or it is perforated, an indirect or direct pulp caping may be indicated if the pulp is healthy, with no inflammation. Bioactive materials such as Biodentine (Biodentine ${ }^{\mathrm{TM}}$ Septodont, Saint-Maurs-des-fosses, France) or MTA (ProRoot MTA, Dentsply Sirona Endodontics, Tulsa Dental, USA) have several advantages including bond strength, short setting time, antibacterial effect and biocompatibility (50-54). Moreover, they help with the formation of reparative dentine, cement and encourage the differentiation of odontoblasts $(55,56)$.

Bioactive materials, such as Biodentine (Septodont), Endosequence Root Repair Material (Brasseler) can replace the use of GI or resin-modified glass ionomer (RMGI) in order to restore the ECR sub-gingival cavity. Although GI and RMGI have certain advantages such as biocompatibility, fluoride release and chemical adhesion to dentine, they do not ensure bone and cement regeneration (57-60). These bioactive restorative materials have the ability to regenerate the cement, the PDL and the bone around them $(50,61)$. If the ECR tooth that is being restored is not located in an area of aesthetic interest, the entire cavity can be restored with bioactive materials; in cases where the aesthetic prevails, 3-4 mm can be removed from the placed material and the remaining cavity can be restored with GI and/or adhesive composite resin (46). 
As stated above, endodontic treatment may be needed if the ECR tooth presents with symptoms of irreversible pulpitis, pulp necrosis and/or apical periodontitis and/or the ECR lesion has perforated the root canal walls. As a technique, it is recommended to initially identify, negotiate and shape the root canal, and then to block the canal space using a suitable gutta-percha cone in order to maintain it patent during the restoration of the ECR lesion. After complete restoration of the defect (as described above), endodontic treatment may be completed. In this way, the risk of blocking the root canal is limited (62).

As an alternative to surgical approach, orthodontic extrusion can be performed in order to access the ECR lesions located far below the gingival margin (38).

Internal approach. When the ECR lesion has perforated or is close to perforate the root canal, and an external repair is not a viable solution, as there is no optimal access, the treatment option is an internal approach. The internal repair technique requires endodontic treatment and subsequent restoration of the ECR cavity (46).

When small entry points are located apically from the epithelial attachment, an internal repair is recommended. An internal repair, meaning endodontic treatment as a first step, can assess teeth with minor communications between the root canal space and the resorptive lesion. After the endodontic treatment is complete, longneck burs and/or ultrasonic tips can be used under the surgical microscope, in order to extend the access cavity so that the ECR defect is included $(46,63)$.

In cases with larger ECR resorptive defects, the endodontic access cavity should be realized with longneck burs and/or ultrasonic instruments in order that both the root canal and the defect are accessed (46).

In order to decontaminate the endodontic system, sodium hypochlorite can be used as the main irrigant. Moreover, sodium hypochlorite can dissolve the granulomatous tissue and can achieve hemostasis. Then, a calcium hydroxide paste, which has an alkaline $\mathrm{pH}$, may be placed in the root canal to stimulate the coagulation necrosis and to inhibit the osteoclastic action, whereas the osteoblastic one occurs $(64,65)$. For the protection of the PDL, a polytetrafluoroethylene tape may be compacted between the tooth and the adjacent tissues. Endodontic treatment may then be finalized.

For restoration of the resulting cavity, materials such GI and/or composite resins may be used. Salzano and Tirone effectively treated four cases of ECR using MTA or Biodentine (66). After the removal of granulomatous tissue through the access cavity and the root canals, the defects were repaired with MTA or Biodentine (66). Applying bioactive materials on the ECR cavities will result in an alkaline $\mathrm{pH}$ that may impede the osteoclastic function of any remaining ECR tissues, reducing the risk of recurrence (67).

\section{Conclusions}

External cervical resorption (ECR) still remains a relatively uncommon pathology which leads to the loss of hard dental tissues due to osteoclastic activity. Its multifactorial etiology being poorly understood, more research is needed to establish the cause-and-effect relationship of all the etiological factors.
The improved radiographic detection using the cone-beam computed tomography (CBCT) allows a precise diagnosis, a more accurate classification of the lesion and leads to a more predictable treatment plan for the benefit of the patients.

\section{Acknowledgements}

Not applicable.

\section{Funding}

No funding was received.

\section{Availability of data and materials}

The authors confirm that all the information provided in this review is documented by relevant references.

\section{Authors' contributions}

RMTN, LMN and MP were strongly involved in the literature review and in writing the manuscript. STN performed the radiological analysis and prepared the images. LCR carefully revised and reviewed the paper in light of the literature data. All authors read and approved the final version of the manuscript for publication.

\section{Ethics approval and consent to participate}

Not applicable.

\section{Patient consent for publication}

The patient provided consent for publication.

\section{Competing interests}

The authors declare that they have no competing interests.

\section{References}

1. Patel S, Kanagasingam S and Pitt Ford T: External cervical resorption: A review. J Endod 35: 616-625, 2009.

2. Patel S, Mavridou AM, Lambrechts P and Saberi N: External cervical resorption-part 1: Histopathology, distribution and presentation. Int Endod J 51: 1205-1223, 2018.

3. Patel S and Ford TP: Is the resorption external or internal? Dent Update 34: 218-20, 222, 224-6, 229, 2007.

4. Patel S and Saberi N: The ins and outs of root resorption. $\mathrm{Br}$ Dent J 224: 691-699, 2018.

5. Heithersay GS: Clinical, radiologic, and histopathologic features of invasive cervical resorption. Quintessence Int 30: 27-37, 1999.

6. Tronstad L: Root resorption-etiology, terminology and clinical manifestations. Endod Dent Traumatol 4: 241-252, 1998.

7. Mavridou AM, Hauben E, Wevers M, Schepers E, Bergmans L and Lambrechts P: Understanding external cervical resorption in vital teeth. J Endod 42: 1737-1751, 2016.

8. Heithersay GS: Invasive cervical resorption. Endod Topics 7: 73-92, 2004.

9. European Society of Endodontology (ESE) developed by: Patel S, Lambrechts P, Shemesh H and Mavridou A: European society of endodontology position statement: External cervical resorption. Int Endod J 51: 1323-1326, 2018.

10. Andreasen JO and Andreasen FM: Textbook and Color Atlas of Traumatic Injuries to the Teeth. 4th edition. Blackwell Munksgaard, Copenhagen, pp1358-1381, 2007. 
11. Kandalgaonkar SD, Gharat LA, Tupsakhare SD and Gabhane MH: Invasive cervical resorption: A review. J Int Oral Health 5: $124-130,2013$

12. Knowles HJ and Athanasou NA: Canonical and non-canonical pathways of osteoclast formation. Histol and Histopathol 24: 337-346, 2009.

13. Mavridou AM, Pyka G, Kerckhofs G, Wevers M, Bergmans L, Gunst V, Huybrechts B, Schepers E, Hauben E and Lambrechts P A novel multimodular methodology to investigate external cervical tooth resorption. Int Endod J 49: 287-300, 2016.

14. Wedenberg C and Lindskog S: Evidence for a resorption inhibitor in dentin. Scand J Dent Res 95: 205-211, 1987.

15. Mavridou AM, Hauben E, Wevers M, Schepers E, Bergmans L and Lambrechts P: Understanding external cervical tooth resorption patterns in endodontically treated teeth. Int Endod J 50: 1116-1133, 2017.

16. Rotondi O, Waldon P and Kim SG: The disease process, diagnosis and treatment of invasive cervical resorption: A review. Dent J (Basel) 8: 64, 2020.

17. Heithersay GS: Invasive cervical resorption: An analysis of potential predisposing factors. Quintessence Int 30: 83-95, 1999.

18. Patel S, Foschi F, Mannocci $F$ and Patel K: External cervical resorption: A three-dimensional classification. Int Endod J 51: 206-214, 2018

19. Mavridou AM, Bergmans L, Barendregt D and Lambrechts P: Descriptive analysis of factors associated with external cervical resorption. J Endod 43: 1602-1610, 2017.

20. Gunst V, Mavridou A, Huybrechts B, Van Gorp G, Bergmans L and Lambrechts P: External cervical resorption: An analysis using cone beam and microfocus computed tomography and scanning electron microscopy. Int Endod J 46: 877-887, 2013.

21. Fuss Z, Tsesis I and Lin S: Root resorption-diagnosis, classification and treatment choices based on stimulation factors. Dent Traumatol 19: 175-182, 2003.

22. Alqerban A, Jacobs R, Lambrechts P, Loozen G and Willems G: Root resorption of the maxillary lateral incisor caused by impacted canine: A literature review. Clin Oral Investig 13 247-255, 2009.

23. Hadler-Olsen S,Pirttiniemi P,KerosuoH,BolstadLimchaichana N, Pesonen P, Kallio-Pulkkinen S and Lähdesmäki R: Root resorptions related to ectopic and normal eruption of maxillary canine teeth-A 3D study. Acta Odontol Scand 73: 609-615, 2015.

24. Friedman S, Rotstein I, Libfield H, Stabholz A and Heling I: Incidence of external root resorption and esthetic results in 58 bleached pulpless teeth. Endod Dent Traumatol 4: 23-26, 1988.

25. Attin T, Paque F, Ajam F and Lennon AM: Review of the current status of tooth whitening with the walking bleach technique. Int Endod J 36: 313-329, 2003.

26. Heithersay GS, Dahlstrom SW and Marin PD: Incidence of invasive cervical resorption in bleached root-filled teeth. Aust Dent J 39: 82-87, 1994.

27. Reiter AM, Lewis JR and Okuda A: Update on the etiology of tooth resorption in domestic cats. Vet Clin North Am Small Anim Pract 35: 913-942, 2005.

28. Reiter AM and Mendoza KA: Feline odontoclastic resorptive lesions an unsolved enigma in veterinary dentistry. Vet Clin North Am Small Anim Pract 32: 791-837, 2002.

29. Von Arx T, Schawalder P, Ackermann M and Bosshardt DD: Human and feline invasive cervical resorptions: The missing link?-Presentation of four cases. J Endod 35: 904-913, 2009.

30. Gunst V, Huybrechts B, De Almeida Neves A, Bergmans L, Van Meerbeek B and Lambrechts P: Playing wind instruments as a potential aetiologic cofactor in external cervical resorption: Two case reports. Int Endod J 44: 268-282, 2011.

31. Irinakis E, Aleksejuniene J, Shen Y and Haapasalo M: External cervical resorption: A retrospective case-control study. J Endod 46: 1420-1427, 2020.

32. Utting JC, Robins SP, Brandao-Burch A, Orriss IR, Behar J and Arnett TR: Hypoxia inhibits the growth, differentiation and bone-forming capacity of rat osteoblasts. Exp Cell Res 312: 1693-1702, 2006.

33. Arnett TR: Acidosis, hypoxia and bone. Arch Biochem Biophys 503: 103-109, 2010.

34. Arnett TR, Gibbons DC, Utting JC, Orriss IR, Hoebertz A, Rosendaal M and Meghji S: Hypoxia is a major stimulator of osteoclast formation and bone resorption. J Cell Physiol 196: 2-8, 2003.

35. Patel K, Mannocci F and Patel S: The assessment and management of external cervical resorption with periapical radiographs and cone-beam computed tomography: A clinical study. J Endod 42: 1435-1440, 2016
36. AAE and AAOMR joint position statement: Use of cone beam computed tomography in endodontics update. J Endod 41: 1393-1396, 2015.

37. Patel S, Durack C, Abella F, Roig M, Shemesh H, Lambrechts P and Lemberg K; European Society of Endodontology: European society of endodontology position statement: The use of CBCT in endodontics. Int Endod J 47: 502-504, 2014.

38. Schwartz RS, Robbins JW and Rindler E: Management of invasive cervical resorption: Observations from three private practices and a report of three cases. J Endod 36: 1721-1730, 2010.

39. Bergmans L, Van Cleynenbreugel J, Verbeken E, Wevers M, Van Meerbeek B and Lambrechts P: Cervical external root resorption in vital teeth, X-ray microfocus-tomographical and histopathological case study. J Clin Periodontol 29: 580-585, 2002.

40. Liang H, Burkes EJ and Frederiksen NL: Multiple idiopathic cervical root resorption: Systematic review and report of four cases. Dentomaxillofac Radiol 32: 150-155, 2003.

41. Patel S, Durack C, Abella F, Shemesh H, Roig M and Lemberg K: Cone beam computed tomography in endodontics-a review. Int Endod J 48: 3-15, 2015.

42. Patel S, Dawood A, Wilson R, Horner K and Mannocci F: The detection and management of root resorption lesions using intraoral radiography and cone beam computed tomography-an in vivo investigation. Int Endod J 42: 831-838, 2009

43. Vaz de Souza D, Schirru E, Mannocci F, Foschi F and Patel S: External cervical resorption: A comparison of the diagnostic efficacy using 2 different cone-beam computed tomographic units and periapical radiographs. J Endod 43: 121-125, 2017.

44. Rodriguez G, Abella F, Duran-Sindreu F, Patel S and Roig M: Influence of cone-beam computed tomography in clinical decision making among specialists. J Endod 43: 194-199, 2017.

45. Rodriguez G, Patel S, Duran-Sindreu F, Roig M and Abella F: Influence of cone-beam computed tomography on endodontic retreatment strategies among general dental practitioners and endodontists. J Endod 43: 1433-1437, 2017.

46. Patel S, Foschi F, Condon R, Pimentel T and Bhuva B: External cervical resorption: Part 2-management. Int Endod J 51: 1224-1238, 2018.

47. Heithersay GS: Treatment of invasive cervical resorption: An analysis of results using topical application of trichloroacetic acid, curettage, and restoration. Quintessence Int 30: 96-110, 1999.

48. Frank AL and Torabinejad M: Diagnosis and treatment of extra-canal invasive resorption. J Endod 24: 500-504, 1998.

49. Heithersay GS and Wilson DF: Tissue responses in the rat to trichloroacetic acid-an agent used in the treatment of invasive cervical resorption. Aust Dent J 33: 451-461, 1988.

50. Prati $\mathrm{C}$ and Gandolfi MG: Calcium silicate bioactive cements: Biological perspectives and clinical applications. Dent Mater 31: 351-370, 2015.

51. Grech L, Mallia B and Camilleri J: Invesitigation of the physical properties of tricalcium silicate cement-based root-end filling materials. Dent Mater 29: e20-e28, 2013.

52. Guo YJ, Du TF, Li HB, Shen Y, Mobuchon C, Hieawy A, Wang ZJ, Yang Y, Ma J and Haapasalo M: Physical properties and hydration behavior of a fast-setting bioceramic endodontic material. BMC Oral Health 16: 23, 2016.

53. Torabinejad $\mathbf{M}$ and Parirokh $\mathrm{M}$ : Mineral trioxide aggregate: $\mathrm{A}$ comprehensive literature review-part II: Leakage and biocompatibility investigations. J Endod 36: 190-202, 2010.

54. Parirokh M and Torabinejad M: Mineral trioxide aggregate: A comprehensive literature review-part I: Chemical, physical, and antibacterial properties. J Endod 36: 16-27, 2010.

55. Torabinejad M, Parirokh M and Dummer PMH: Mineral trioxide aggregate and other bioactive endodontic cements: An updated overview-part II: Other clinical applications and complications. Int Endod J 51: 284-317, 2018.

56. Parirokh M, Torabinejad M and Dummer PMH: Mineral trioxide aggregate and other bioactive endodontic cements: An updated overview-part I: Vital pulp therapy. Int Endod J 51: 177-205, 2018.

57. Rajasekharan S, Martens LC, Cauwels RG and Verbeeck RM: Biodentine material characteristics and clinical applications: A review of the literature. Eur Arch Paediatr Dent 15: 147-158, 2014.

58. Hasan AMHR, Sidhu SK and Nicholson JW: Fluoride release and uptake in enhanced bioactivity glass ionomer cememt ('glass carbomer $^{\mathrm{TM}}$ ') compared with conventional and resin-modified glass ionomer cements. J Appl Oral Sci 27: e20180230, 2019.

59. Sidhu SK and Nicholson JW: A review of glass-ionomer cements for clinical dentistry. J Funct Biomater 7: 16, 2016. 
60. Santamaria MP, Ambrosano GM, Casati MZ, Nociti Júnior FH, Sallum AW and Sallum EA: Connective tissue graft plus resin-modified glass ionomer restoration for the treatment of gingival recession associated with non-carious cervical lesion: A randomized-controlled clinical trial. J Clin Periodontol 36: 791-798, 2009.

61. Parirokh M and Torabinejad M: Mineral trioxide aggregate: A comprehensive literature review-Part III: Clinical applications, drawbacks, and mechanism of action. J Endod 36: 400-413, 2010

62. Patel S, Durack C and Ricucci D: Root resorption. In: Pathways of the pulp. 11th edition. Hargreaves KM and Berman LH (eds). Elsevier, St. Louis, pp660-683, 2016.

63. Frank AL: External-internal progressive resorption and its nonsurgical correction. J Endod 7: 473-476, 1981.

64. Aeinehchi M, Eslami B, Ghanbariha M and Saffar AS: Mineral trioxide aggregate (MTA) and calcium hydroxide as pulp-capping agents in human teeth: A preliminary report. Int Endod J 36: 225-231, 2003
65. Narita H, Itoh S, Imazato S, Yoshitake F and Ebisu S: An explanation of the mineralization mechanism in osteoblasts induced by calcium hydroxide. Acta Biomater 6: 586-590, 2010.

66. Salzano S and Tirone F: Conservative nonsurgical treatment of class 4 invasive cervical resorption: A case series. J Endod 41: 1907-1912, 2015

67. Arnett TR: Extracellular $\mathrm{pH}$ regulates bone cell function. J Nutr 138: 415S-418S, 2008.

(i) (9) This work is licensed under a Creative Commons Attribution-NonCommercial-NoDerivatives 4.0 International (CC BY-NC-ND 4.0) License. 